\begin{tabular}{|c|c|}
\hline \multirow{3}{*}{ 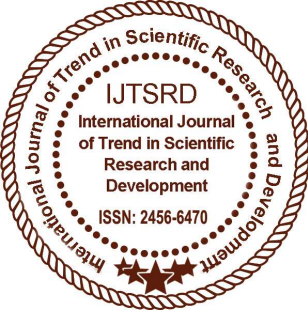 } & $\begin{array}{l}\text { International Journal of Trend in Scientific } \\
\text { Research and Development (IJTSRD) }\end{array}$ \\
\hline & International Open Access Journal \\
\hline & ISSN No: 2456 - 6470 | www.ijtsrd.com | Volume - 2 | Issue - 4 \\
\hline
\end{tabular}

\title{
NOSQL: A Very Dynamic Approach for Managing Big Data
}

\author{
Assira Amin \\ M.Tech Scholar, Department of \\ CSE'Sat Kabir Institute of \\ Technology \& Management, \\ Haryana, India
}

\author{
Mudasir Ahmed Muttoo \\ Assistant Professor, Department of \\ CSE'SSM College of Engineering \\ \& Technology, \\ Kashmir, India
}

\author{
Kirti Bhatia \\ Assistant Professor, Department of \\ CSE' Sat Kabir Institute of \\ Technology \& Management, \\ Haryana, India
}

\begin{abstract}
Database Management System is very dynamic and popular subject in Computer Science and Information Technology. Modern society is based on 'e' concept means every task is achieved by interactive applications like e-commerce applications and the production of data is more and more. The present digital world becomes more complex with Big Data means the volume, velocity and variety of data. The Big Data is a global system and is considered a data collection that increases so large and can't be managed efficiently by traditional databases management systems. The aim of this paper is to identify the new data management strategies and how to tackle Big Data through a new breed of databases commonly known as NOSQL databases.
\end{abstract}

Keywords: NOSQL; RDBMS; BIG Data; Business Intelligence; $C A P$

\section{INTRODUCTION}

Database management system is a methodology for storing and accessing data efficiently. Various models have been proposed by experts time by time for data management and the relational model is the most popular and adoptable by professionals. Modern applications based on social networking and business intelligence changes the data store strategy to a great extent. The traditional databases are not efficient for handling the Big Data that is the management of data in terms of volume, variety and velocity of data. NOSQL databases are very good alternative for managing the Big Data. NOSQL is a group of nonrelational database management systems, not mostly based on tables and do not use SQL for data manipulation. NOSQL databases are very efficient specially handling huge volumes of data. NOSQL is based on distributed concept for huge data storage and supports parallel processing of data across a large number of commodity servers.

\section{Big Data characteristics}

Big Data is not only size but also includes the variety and velocity of data.

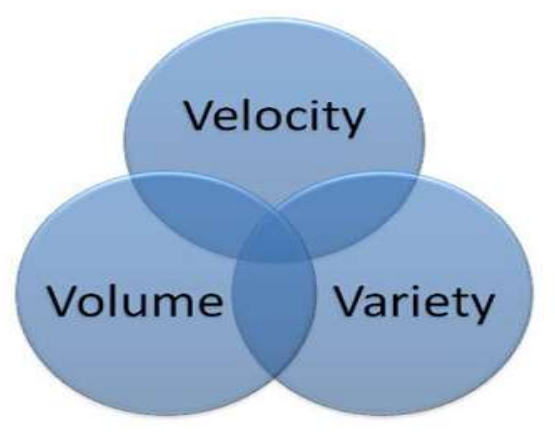

The volume of data in an organization grow regardless of the size because there is a need to store data of all varieties like medical data, financial data etc. Today data of organisations are in the terabyte range but soon can move to petabytes or exabytes. Data in an enterprise also travel from various sources in different ways like use of sensors, deployment of 
smart devices and the most important social networking. The enterprise data not only contain structured data but also un-structured and semistructured data thus making enterprise data more and more complex.

\section{Structured Data}

Structured one represents data that is grouped into a relational format means in terms of rows and columns within a database and respond to the simple queries based on parameters and operational requirements.

\section{Semi-structured Data}

Semi-structured data is form of structured one and not validate against a fixed schema. The data is self describing and contains tags to enforce hierarchies of fields and records.

\section{Un-Structured Data}

This type includes data formats which are not indexed into relational tables for querying like audio, video and image files.

The velocity is also one of the important characteristic of Big Data. Velocity simple means how fast the data appear, stored and retrieved. The value of Big Data is extracted by enterprises only after tackling the $3 \mathrm{Vs}$ means volume, variety and velocity of data.

\section{NOSQL for Big Data}

DataStax is the leading provider of efficient database technologies and services and is very famous for providing best options for handling the Big Data. The foundation for DataStax for handling Big Data is the power of Apache Cassandra and is the only NOSQL database technology that truly handles the modern trend of data management commonly known as Big Data. The key technical aspects of Cassandra NOSQL database technology are described as under:

- Cassandra can store data even in petabytes and support thousands of concurrent users per second with easiness.

- Supports peer-to-peer design that results in no single point of failure for any process of database.

- Cassandra is based on location independent concept means data can be written and read from anywhere.
- RDBMS like data durability and protection because support tunable data consistency.

- Cassandra supports flexible design means support all Big Data all formats including structured, semi-structured and un-structured.

- The replication is very simple and supports multi data center and is very efficient for cloud environment.

- Very comfortable database for leading programming languages.

- Cassandra runs efficiently on commodity hardware means no need of additional hardware.

Theses aspects make Cassandra the best choice for enterprises specially for handling Big Data.

\section{CONCLUSION}

The database is a very important part for any application. The modern applications based on social networking and business intelligence change the data storage concept to a great extent. The volume of data in organizations grows rapidly up to petabytes or even exabytes and is very difficult for traditional database systems to handle. The NOSQL is a new open source, distributed data storage concept that is very efficient in terms of handling the huge volume of data. The Big Data refers not only the volume of data but also the variety and the velocity of data.

\section{REFERENCES}

1. AmeyaNayak, Anil Poriya, DikshayPoojary "Type of NOSQL Databases and Its Comparison with Relational Databases," International Journal of Applied Information Systems, Vol.5, No. 4, 2013

2. A B M Moniruzzaman, Syed AkhterHossain "NOSQL Database: New Era of Databases for Big Data Analytics-Classification, Characteristics and Comparison," International Journal of Database Theory and Application, Vol. 6, No. 4, 2013

3. Dr K Chitra, BJeevaRani "Study on Basically Available, Scalable and Eventually Consistent NOSQL Databases," International Journal of Advanced Research and Software Engineering, Vol. 3, 2013

4. InduArora, DrAnu Gupta "Cloud Databases: A Paradigm Shift in Databases," International Journal of Computer Sciences, Vol. 9, No. 3, 2012

5. J M Tauro, Aravindh S, Shreeharsha A.B "Comparative Study of the New Generation, 
Agile, Scalable, High Performance NOSQL Databases," International Journal of Computer Applications, Vol. 48, No. 20, 2012

6. NishthaJatana, SahilPuri, MehakAhuja, IshitaKathuria, DishantGosain "A Survey of Relational and Non-Relational Database," International Journal of Engineering Research \& Technology, Vol. 1, 2012

7. Paolo Atzeni, Christian S. Jenson, Orsi, Sudha Ram, Tanca, Torlone "The Relational Model is
Dead, SQL is Dead, and I don't feel so good myself, "SIGMOD, Vol. 42, No. 2, 2013

8. DataStax Corporation "NOSQL in the Enterprise", 2011

9. Forsyth Communications "For Big Data Analytics There's Such Thing as Too Big", 2012

10. Oracle "Big Data for the Enterprise," 2013

11. Oracle "Hadoop and NOSQL Technologies and the Oracle Database, 2011

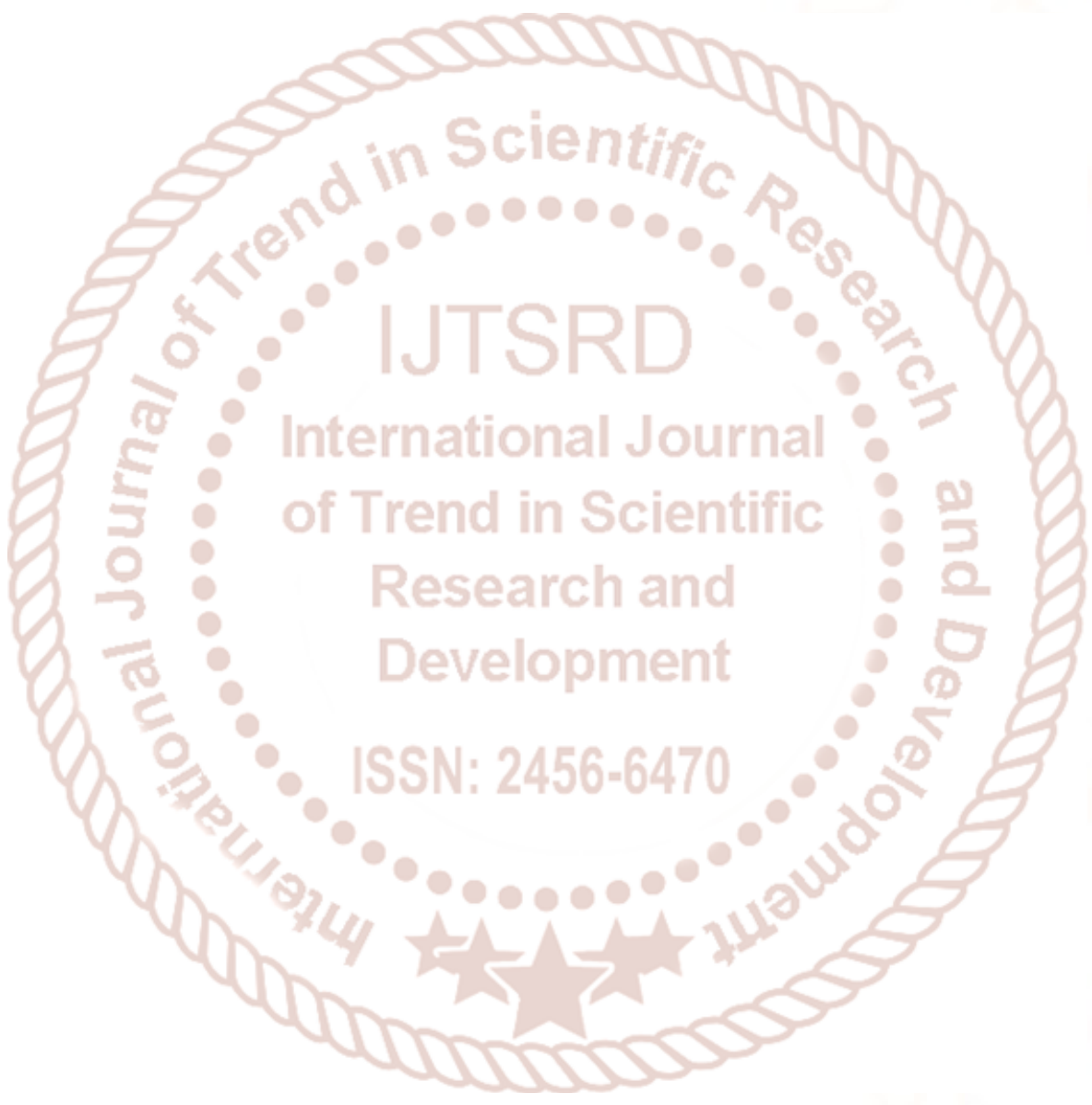

